\section{Auch am Hals ist auf die Wächterlymphknoten-Biopsie Verlass}

\author{
Mit der Biopsie von Wächterlymphknoten lassen sich zuverlässig okkulte \\ Metastasen am Hals von Patienten mit Mundhöhlenkarzinomen ausschließen. \\ Allerdings ist die Rate falsch-negativer Ergebnisse grenzwertig hoch.
}

\begin{abstract}
$\mathrm{R}$ und jeder dritte Patient mit Mundhöhlenkarzinom im Stadium I/II hat radiologisch nicht nachweisbare zervikale Metastasen. Die Heilungschance sinkt dadurch um die Hälfte. Bei einem Metastasierungsrisiko von über $20 \%$ schlägt man den Patienten meist eine elektive Neck-Dissection vor, d.h. fast $80 \%$ unterziehen sich dieser Prozedur unnötigerweise. Die Entnahme von Wächterlymphknoten (SLN) wurde deshalb in der multizentrischen, prospektiven europäischen SENT-Studie getestet, deren 3-Jahres-Ergebnisse nun vorliegen. Bei den 415 Patienten hatte die radiologische Diagnostik einen Tumor im Stadium T1-2N0 ergeben. Nach Injektion eines Technetium-Nanokolloids ließ sich bei 99,5\% der Patienten ein SLN identifizieren. In 94 von 415 Fällen
\end{abstract}

blieb. Allerdings liege die Rate falschnegativer Ergebnisse mit $14 \%$, an der Grenze des Akzeptablen“. Zwar ähnle die Rate jener, die von Melanompatienten bekannt sei, sie sei aber doppelt so hoch wie bei Patientinnen mit Mammakarzinom. „7\% sollten unser Ziel sein".

Andererseits war nur bei 8 von 15 der fälschlich als nodal negativ eingestuften Patienten ein rettender Eingriff möglich, und mit der SLN-Methode konnten 7 positive Knoten kontralateral zur Tumorlage ausgemacht werden, die man bei der üblichen ipsilateralen NeckDissection nicht erkannt hätte.

Fazit: Die SLN-Biopsie ist auch bei Patienten ohne okkulte zervikale Lymphknotenmetastasen relativ zuverlässig. Noch lässt die Sensitivität zu wünschen übrig, aber sie könnte durch verbesserte Technik und mehr Erfahrung gesteigert werden.

Dr. Robert Bublak $94 \%$ - jeweils nach drei Jahren.

Den Hauptnutzen der SLN-Methode sehen die Forscher darin, dass $71 \%$ der Patienten eine Neck-Dissection erspart

\title{
Nasenfilter schützt vor allergischen Rhinitissymptomen
}

\section{Pollenundurchlässige Nasenfilter bieten einen kausalen Therapieansatz bei saisonaler allergischer Rhinitis. Eine Proof-of-Concept-Studie zur Effektivität, Verträglichkeit und Akzeptanz verlief zufriedenstellend.}

$\mathrm{M}$ echanische Schutzsysteme zur Vermeidung des Allergenkontakts mit der Nasenschleimhaut, bisher überwiegend in Form von Atemmasken oder Nasensalben angeboten, haben sich bei Patienten mit saisonaler allergischer Rhinitis nicht recht durchsetzen können. Neue, intranasale Nasenfilter könnten das ändern. Sie stören die Träger offensichtlich kaum und erwiesen sich in ersten Untersuchungen in Pollenkammerversuchen als brauchbar. Zusätzlich erfolgte die Erprobung unter natürlichen Bedingungen während der Pollensaison. Man bediente sich dabei einer „Tag-imPark-Studie“. Anders als bei multizent- rischen Studien über die gesamte Pollensaison erlauben Parkstudien eine genauere Assoziation zwischen Pollenbelastung und Symptomatik.

Einbezogen in die monozentrische, doppelblinde, placebokontrollierte Studie waren 65 erwachsene Gräserpollenallergiker, die entweder die neuen $\mathrm{Na}$ senfilter oder Placebofilter über zwei Tage im Cross-over-Design während der Hauptgräserpollensaison trugen. Das Einsetzen der Filter sollte möglichst bei noch geringer Symptombelastung erfolgen. Primäres Studienziel war der Unterschied zwischen beiden Gruppen beim nasalen Gesamtsymptomenscore
(TNSS) mit insgesamt 13 Einzelitems. Hier ergab sich ein signifikanter Unterschied zugunsten der Nasenfilter ( $\mathrm{p}=$ 0,03 ), auch der Unterschied bei den Maximal-TNSS zwischen beiden Gruppen war signifikant $(p=0,03)$, entsprechend einem relativen Rückgang der Beschwerden von $40 \%$ und $43 \%$. Überdurchschnittlich gut schützten die Filter vor Niesen und laufender Nase. Sogar das Augentränen ließ bei den Probanden mit Verumfilter deutlich nach. Schwerwiegende Nebenwirkungen wurden nicht beschrieben, die Akzeptanz war gut.

Fazit: Ein neuer Nasenfilter reduzierte im Vergleich zu einem Placebofilter die Symptome einer allergischen Gräserpollenrhinitis signifikant.

Dr. Barbara Kreutzkamp

Kenney et al. Preventive effect of nasal filters on allergic rhinitis: A randomized, double-blind, placebo-controlled crossover park study. J Allergy Clin Immunol 2015; 136: 1566-72 